\title{
STRICT FEASIBILITY OF GENERALIZED COMPLEMENTARITY PROBLEMS
}

\author{
Y. R. HE ${ }^{\longleftarrow}$ and K. F. NG \\ (Received 17 November 2004; revised 15 February 2005) \\ Communicated by A. Rubinov
}

\begin{abstract}
The existence of strictly feasible points is shown to be equivalent to the boundedness of solution sets of generalized complementarity problems with stably pseudomonotone mappings. This generalizes some known results in the literature established for complementarity problems with monotone mappings.
\end{abstract}

2000 Mathematics subject classification: primary 90C $33,49 \mathrm{~J} 40$.

Keywords and phrases: generalized complementarity problem, stably pseudomonotone mapping. strict feasibility, upper hemicontinuous set-valued mapping.

\section{Introduction and preliminaries}

Throughout this note (unless explicitly stated otherwise), let $K$ denote a nonempty closed convex subset of $\mathbb{R}^{n}$ and $F$ denote a set-valued mapping from $K$ into $\mathbb{B}^{n}$. We make the blanket assumption that the mapping $F$ is upper hemicontinuous on $K$ with nonempty compact convex values. The generalized variational inequality problem, denoted by $\operatorname{GVIP}(F, K)$, is to find vectors $x \in K$ and $x^{*} \in F(x)$ such that

$$
\left\langle x^{*}, y-x\right\rangle \geq 0 \text { for all } y \in K,
$$

where $\langle\cdot, \cdot\rangle$ denotes the usual inner product in $\mathbb{R}^{n}$. If $K$ is a nonempty closed convex cone in $\mathbb{R}^{n}$, then the $\operatorname{GVIP}(F, K)$ is the same as the generalized complementarity problem $\operatorname{GCP}(F, K)$ (see [8]): to find $x \in K$ and $x^{*} \in F(x)$ such that $x^{*} \in K^{+}$and

The research was supported by an earmarked grant from the Research Grant Council of Hong Kong. Research of the first author was supported by Sichuan Youth Science and Technology Foundation (06ZQ026-13) and grant SZD0406 from Sichuan Province.

(C) 2006 Australian Mathematical Society 1446-7887/06 \$A2.00+0.00 
$\left\langle x^{*}, x\right\rangle=0$, where $K^{+}$is the positive polar cone of $K$. Assume that the interior of $K^{+}$is nonempty, that is, $\operatorname{int}\left(K^{+}\right) \neq \emptyset$. We say that the problem $\operatorname{GCP}(F, K)$ is strictly feasible if $F(K) \cap \operatorname{int}\left(K^{+}\right) \neq \emptyset$.

A mapping $F$ is said to be upper semicontinuous on $K$ if for any open set $V$, $\{y \in K \mid F(y) \subset V\}$ is open relative to $K ; F$ is said to be upper hemicontinuous on $K$ if the restriction of $F$ to each line segment of $K$ is upper semicontinuous. Recall also that $F$ is said to be pseudomonotone on $K$ if for all $x_{1}, x_{2} \in K, x_{1}^{*} \in F\left(x_{1}\right)$ and $x_{2}^{*} \in F\left(x_{2}\right),\left\langle x_{1}^{*}, x_{2}-x_{1}\right\rangle \geq 0$ implies $\left\langle x_{2}^{*}, x_{2}-x_{1}\right\rangle \geq 0$. A pseudomonotone mapping $F$ is said to be stably pseudomonotone on $K$ with respect to a set $U \subset \mathbb{R}^{n}$ if the mapping $F(\cdot)-u$ is pseudomonotone on $K$ for every $u \in U$. For a nonempty convex set $K$, barr $(K)$ denotes its barrier cone [6] and $K^{+}$denotes the set $\left\{x^{*} \in \mathbb{R}^{n} \mid\left\langle x^{*}, x\right\rangle \geq 0\right.$ for all $\left.x \in K\right\}$. A function $h: \mathbb{R}^{n} \rightarrow \mathbb{R} \cup\{+\infty\}$ is said to be proper if $\operatorname{dom}(h)$ defined as the set $\left\{x \in \mathbb{R}^{n} \mid h(x)<+\infty\right\}$ is nonempty; and it is said to be level-bounded on $K$ if the set $\{x \in K \mid h(x) \leq r\}$ is bounded for every $r \in \mathbb{R}$.

In Section 2, we prove the following theorem.

THEOREM 2.3. Let $K$ be a nonempty closed convex cone with $\operatorname{int}\left(K^{+}\right)$nonempty. Assume that there exists a sequence $\left\{u_{m}\right\} \subset \operatorname{int}\left(K^{+}\right)$such that (a) $\lim _{m \rightarrow \infty} u_{m}=0$ and $\left\{u_{m}\right\}$ is decreasing with respect to $K^{+}$; (b) the mapping $F(\cdot)-u_{m}$ is pseudomonotone on $K$ for each $m \in \mathbb{N}$. Then $\operatorname{GCP}(F, K)$ is strictly feasible if and only if its solution set is nonempty and bounded.

Theorem 2.3 generalizes [5, Theorem 4] and [1, Corollary 1]. The former assumed $F$ is maximal monotone while the latter assumed $F$ is monotone and singlevalued mapping, and $K=\mathbb{R}_{+}^{n}$. Example 2 in [4] shows that Theorem 2.3 genuinely generalizes the aforementioned results.

LEMMA 1.1. Let $K$ be a nonempty closed convex subset of $\mathbb{R}^{n}$ and let

$$
\operatorname{int}(\operatorname{barr}(K)) \neq \emptyset \text {. }
$$

Then $e \in \operatorname{int}(-\operatorname{barr}(K))$ if and only if the set $E(r):=\{x \in K \mid\langle e, x\rangle \leq r\}$ is bounded for every $r \in \mathbb{R}$.

PROOF. Define a function $f(x):=\langle e, x\rangle+I_{K}(x)$, where $I_{K}(x)$ denotes the indicator function of $K$. Then $f$ is proper convex lower semicontinuous and $E(r)$ is the level set of $f$. The conclusion now follows from [6, Corollaries 8.7.1 and 14.2.2].

We need to introduce an additional function. For $x \in K$ and $u \in \mathbb{R}^{n}$,

$$
\hat{f}(x, u)=\sup \left\{\frac{\left\langle y^{*}-u, x-y\right\rangle}{\max \left\{\left\|y^{*}\right\|, 1\right\} \max \{\|y\|, 1\}} \mid y^{*} \in F(y), y \in K\right\},
$$

The function $\hat{f}(\cdot, 0)$ is considered in [2]. 


\section{Main results}

PROPOSITION 2.1. Let $K$ be a nonempty closed convex set in $\mathbb{R}^{n}$ and $u \in \mathbb{R}^{n}$ be such that $F(\cdot)-u$ is pseudomonotone on $K$. Then $x \in K$ solves $\operatorname{GVIP}(F-u, K)$ if and only if $\hat{f}(x, u)=0$.

Proof. Assume that $x \in K$ solves $\operatorname{GVIP}(F-u, K)$. Then there exists $x^{*} \in F(x)$ such that $\left\langle x^{*}-u, x-y\right\rangle \leq 0$ for all $y \in K$. Since $F(\cdot)-u$ is pseudomonotone, it follows that $\left\langle y^{*}-u, x-y\right\rangle \leq 0$ for all $y \in K$ and $y^{*} \in F(y)$. This shows that $\hat{f}(x, u) \leq 0$. On the other hand, the definition of $\hat{f}$ implies that $\hat{f}(x, u) \geq 0$ for every $x \in K$. Hence $\hat{f}(x, u)=0$.

Conversely, assume that $\hat{f}(x, u)=0$. Let $y \in K$ and define $y_{t}=x+t(y-x)$ for $t \in(0,1]$. Pick $y_{t}^{*} \in F\left(y_{t}\right)$. Then the definition of $\hat{f}$ implies $\left\langle y_{t}^{*}-u, x-y\right\rangle \leq 0$. Since $F$ is upper hemicontinuous with compact values, we assume without loss of generality that $\left(y_{t}^{*}\right) \rightarrow z^{*} \in F(x)$ as $t \rightarrow 0+$. Thus $\left\langle z^{*}-u, x-y\right\rangle \leq 0$. Since $y \in K$ is arbitrary,

$$
\sup _{y \in K} \inf _{x^{*} \in F(x)}\left\langle x^{*}-u, x-y\right\rangle \leq 0 .
$$

Since $F(x)$ is a nonempty compact convex set and $K$ is a closed convex set, we can apply Sion's minimax theorem [9] to obtain that

$$
\inf _{x^{*} \in F(x)} \sup _{y \in K}\left\langle x^{*}-u, x-y\right\rangle=\sup _{y \in K} \inf _{x^{*} \in F(x)}\left\langle x^{*}-u, x-y\right\rangle \leq 0 .
$$

Since $x^{*} \mapsto \sup _{y \in K}\left\langle x^{*}-u, x-y\right\rangle$ is clearly lower semicontinuous and $F(x)$ is compact, it follows that there exists $x^{*} \in F(x)$ such that $\sup _{y \in K}\left\langle x^{*}-u, x-y\right\rangle \leq 0$, so $x$ solves $\operatorname{GVIP}(F-u, K)$.

LEMMA 2.2. Let $u \in \mathbb{R}^{n}$ be such that $F(\cdot)-u$ is pseudomonotone on $K$ and that $\hat{f}(\cdot, u)$ is level-bounded on $K$. Then there exists $x \in K$ such that $x$ solves $\operatorname{GVIP}(F-u, K)$ and hence $\hat{f}(x, u)=0$.

ProOF. Define $K_{i}=\{x \in K \mid\|x\| \leq i\}, i \in \mathbb{N}$, and

$$
\hat{f}_{i}(x, u)=\sup \left\{\frac{\left\langle y^{*}-u, x-y\right\rangle}{\max \left\{\left\|y^{*}\right\|, 1\right\} \max \{\|y\|, 1\}} \mid y^{*} \in F(y), y \in K_{i}\right\} .
$$

The boundedness of $K_{i}$ implies that (see [3, Corollary 1]) there exists some $x_{i} \in K_{i}$ that solves $\operatorname{GVIP}\left(F-u, K_{i}\right)$. For some $x_{i}^{*} \in F\left(x_{i}\right)$,

$$
\left\langle x_{i}^{*}-u, x_{i}-y\right\rangle \leq 0, \text { for all } y \in K_{i} .
$$


Then $\hat{f}_{i}\left(x_{i}, u\right)=0$ by Proposition 2.1. We claim that $\left\|x_{i}\right\|<i$ for some $i \in \mathbb{N}$. Otherwise, $\left\|x_{i}\right\|=i$ for each $i$. Note that $\left\{\hat{f}_{i}(x, u)\right\}$ is increasing and pointwise converging on $K$ to $\hat{f}(x, u)$. Since $\hat{f}(x, u)$ is level-bounded on $K$, [7, Corollary 4.12] shows that $\hat{f}_{i_{0}}(x, u)$ is level-bounded on $K$ for some $i_{0} \in \mathbb{N}$. Set

$$
C=\left\{x \in K \mid \hat{f}_{i_{0}}(x, u) \leq 1\right\} \text { and } c=\sup _{x \in C}\|x\| .
$$

Then $C$ is compact and nonempty. If $i>\max \left\{i_{0}, c\right\}$, then $x_{i} \notin C$. Hence $\hat{f}_{i}\left(x_{i}, u\right)>1$ which contradicts $\hat{f}_{i}\left(x_{i}, u\right)=0$. Thus there must exist $j$ such that $\left\|x_{j}\right\|<j$. We claim that $x_{j}$ solves $\operatorname{GVIP}(F-u, K)$. Indeed for each $y \in K$ there exists sufficiently small $t>0$ such that $x_{j}+t\left(y-x_{j}\right) \in K_{j}$. It follows from (2) that $t\left\langle x_{j}^{*}-u, x_{j}-y\right\rangle \leq 0$, and hence $\left\langle x_{j}^{*}-u, x_{j}-y\right\rangle \leq 0$. Thus $x_{j}$ solves $\operatorname{GVIP}(F-u, K)$, and hence $\hat{f}\left(x_{j}, u\right)=0$ by Proposition 2.1.

THEOREM 2.3. Let $K$ be a nonempty closed convex set with $\operatorname{int}\left(K^{+}\right)$nonempty, and suppose that there exists a sequence $\left\{u_{m}\right\} \subset \operatorname{int}\left(K^{+}\right)$decreasing with respect to $K^{+}$ and converging to zero such that $F$ is stably pseudomonotone on $K$ with respect to $\left\{u_{m}\right\}$. Then $F(K) \cap \operatorname{int}(-\operatorname{barr}(K)) \neq \emptyset$ if and only if $\operatorname{GVIP}(F, K)$ has a nonempty and bounded solution set.

Proof. Suppose that $\operatorname{GVIP}(F, K)$ has a nonempty and bounded solution set. By Proposition 2.1, the set $\{x \in K \mid \hat{f}(x, 0)=0\}$ is nonempty and bounded. Hence, [6, Corollary 8.7.1] implies that $\hat{f}(\cdot, 0)$ is level-bounded on $K$. We claim that there exists $m \in \mathbb{N}$ such that $\hat{f}\left(\cdot, u_{m}\right)$ is also level-bounded on $K$. To see this, we define $h_{m}: K \rightarrow \mathbb{R}$ for each $m \in \mathbb{N}$ by $h_{m}(x):=\hat{f}(x, 0)-\left\langle u_{m}, x\right\rangle$. Clearly $\left\{h_{m}\right\}$ is increasing and pointwise converges to $\hat{f}(x, 0)$. By [7, Corollary 4.12], $h_{m}$ is levelbounded on $K$ if $m$ is large enough. Thus our claim will stand if one can show that

$$
h_{m}(x) \leq \hat{f}\left(x, u_{m}\right), \quad \text { for all } x \in K, m \in \mathbb{N} .
$$

To verify (3), let $x \in K$ and $m \in \mathbb{N}$. For any $\varepsilon>0$, the definition of $\hat{f}(x, 0)$ implies that there exist $y \in K$ and $y^{*} \in F(y)$ such that

$$
\hat{f}(x)<\frac{\left\langle y^{*}, x-y\right\rangle}{\max \left\{\left\|y^{*}\right\|, 1\right\} \max \{\|y\|, 1\}}+\varepsilon .
$$

This implies that

$$
\begin{aligned}
\hat{f}(x, 0) & -\hat{f}\left(x, u_{m}\right) \\
\leq & \frac{\left\langle y^{*}, x-y\right\rangle}{\max \left\{\left\|y^{*}\right\|, 1\right\} \max \{\|y\|, 1\}}-\frac{\left\langle y^{*}-u_{m}, x-y\right\rangle}{\max \left\{\left\|y^{*}\right\|, 1\right\} \max \{\|y\|, 1\}}+\varepsilon
\end{aligned}
$$




$$
\begin{aligned}
& =\frac{\left\langle u_{m}, x-y\right\rangle}{\max \left\{\left\|y^{*}\right\|, 1\right\} \max \{\|y\|, 1\}}+\varepsilon \\
& \leq \frac{\left\langle u_{m}, x\right\rangle}{\max \left\{\left\|y^{*}\right\|, 1\right\} \max \{\|y\|, 1\}}+\varepsilon \\
& \leq\left\langle u_{m}, x\right\rangle+\varepsilon,
\end{aligned}
$$

where the last two inequalities hold because $u_{m} \in K^{+}$and $x, y \in K$. Letting $\varepsilon \rightarrow 0+$, it follows that $h_{m}(x)=\hat{f}(x, 0)-\left\langle u_{m}, x\right\rangle \leq \hat{f}\left(x, u_{m}\right)$. Thus (3) holds and $\hat{f}\left(\cdot, u_{m}\right)$ is level-bounded on $K$. Consequently, by virtue of Lemma 2.2, there exists some $x_{m} \in K$ such that $x_{m}$ solves $\operatorname{GVIP}\left(F-u_{m}, K\right)$. This implies that $x_{m}^{*}-u_{m} \in-\operatorname{barr}(K)$ and so $x_{m}^{*} \in \operatorname{int}(-\operatorname{barr}(K))$. Therefore $x_{m}^{*} \in F\left(x_{m}\right) \cap \operatorname{int}(-\operatorname{barr}(K))$. Conversely, suppose that there exist $x_{0} \in K$ and $x_{0}^{*} \in F\left(x_{0}\right)$ such that $x_{0}^{*} \in \operatorname{int}(-\operatorname{barr}(K))$. Define

$$
D:=\left\{x \in K \mid\left\langle x_{0}^{*}, x-x_{0}\right\rangle \leq 0\right\} .
$$

By Lemma 1.1, $D$ is bounded. Let $x \in K \backslash D$. Since $F$ is pseudomonotone, $\left\langle x^{*}, x-x_{0}\right\rangle>0$ for all $x^{*} \in F(x)$. Thus one can apply [3, Corollary 1] to conclude that the solution set of $\operatorname{GVIP}(F, K)$ is nonempty and bounded.

We end this note with two simple examples to show that the condition on stable pseudomonotonicity in Theorem 2.3 cannot be dropped. Let

$$
K=\left\{(x, y) \in \mathbb{R}^{2} \mid x+i y=r \exp (i \theta) \text { with } \theta \in[\pi / 6, \pi / 3] \text { and } r \geq 0\right\} .
$$

EXAMPLE 1. For each $(x, y) \in K$ with $x+i y=r \exp (i \theta)$, define

$$
F(x, y)=r^{8}(\cos (8 \theta-5 \pi / 6), \sin (8 \theta-5 \pi / 6)) .
$$

Then $\operatorname{GCP}(F, K)$ is strictly feasible with unbounded solution set.

EXAMPLE 2. For each $(x, y) \in K$ with $x+i y=r \exp (i \theta)$, define

$$
F(x, y)=r^{6} \sqrt{r}(\cos (13 \theta / 2-3 \pi / 8), \sin (13 \theta / 2-3 \pi / 8)) .
$$

Then $\operatorname{GCP}(F, K)$ has a bounded solution set, but is not strictly feasible.

\section{References}

[1] B. Chen. X. Chen and C. Kanzow, 'A penalized Fischer-Burmeister NCP-function', Math. Program. Ser. A 88 (2000), 211-216.

[2] J.-P. Crouzeix, 'Pseudomonotone variational inequality problems: Existence of solutions', Math. Program. Ser. A 78 (1997), 305-314. 
[3] A. Daniilidis and N. Hadjisavvas, 'Coercivity conditions and variational inequalities', Math. Program. Ser. A 86 (1999), 433-438.

[4] Y. He, 'A relationship between pseudomonotone and monotone mappings', Appl. Math. Lett. 17 (2004), 459-461.

[5] L. McLinden, 'Stable monotone variational inequalities', Math. Program. Ser. B 48 (1990), 303-338.

[6] R. T. Rockafellar, Convex analysis (Princeton University Press, Princeton, N.J., 1970).

[7] R. T. Rockafellar and R. J.-B. Wets, Variational analysis (Springer, Berlin, 1998).

[8] R. Saigal, 'Extension of the generalized complementarity problem', Math. Oper. Res. 1 (1976), 260-266.

[9] M. Sion, 'On general minimax theorems', Pacific J. Math. 8 (1958), 171-176.

Department of Mathematics

Sichuan Normal University

Chengdu, Sichuan

P. R. China

e-mail: yiranhe@ hotmail.com
Department of Mathematics The Chinese University of Hong Kong Shatin, New Territories Hong Kong e-mail: kfng@math.cuhk.edu.hk 